\title{
ON THE ELECTRON-TO-NEUTRAL NUMBER DENSITY RATIO IN THE COMA OF COMET 67P/ CHURYUMOV-GERASIMENKO: GUIDING EXPRESSION AND SOURCES FOR DEVIATIONS
}

\author{
E. Vigren ${ }^{1}$, M. Galand ${ }^{2}$, A. I. Eriksson ${ }^{1}$, N. J. T. Edberg ${ }^{1}$, E. Odelstad ${ }^{1}$, and S. J. Schwartz ${ }^{2}$ \\ ${ }^{1}$ Swedish Institute of Space Physics, Uppsala, Sweden; erik.vigren@irfu.se \\ ${ }^{2}$ Department of Physics, Imperial College London, London, SW7 2AZ, UK \\ Received 2015 June 22; accepted 2015 September 2; published 2015 October 7
}

\begin{abstract}
We compute partial photoionization frequencies of $\mathrm{H}_{2} \mathrm{O}, \mathrm{CO}_{2}$, and $\mathrm{CO}$, the major molecules in the coma of comet 67P/Churyumov-Gerasimenko, the target comet of the ongoing ESA Rosetta mission. Values are computed from Thermosphere Ionosphere Mesosphere Energy and Dynamics/Solar EUV Experiment solar EUV spectra for 2014 August 1, 2015 March 1, and for perihelion (2015 August, as based on prediction). From the varying total photoionization frequency of $\mathrm{H}_{2} \mathrm{O}$, as computed from 2014 August 1 to 2015 May 20, we derive a simple analytical expression for the electron-to-neutral number density ratio as a function of cometocentric and heliocentric distance. The underlying model assumes radial movement of the coma constituents and does not account for chemical loss or the presence of electric fields. We discuss various effects/processes that can cause deviations between values from the analytical expression and actual electron-to-neutral number density ratios. The analytical expression is thus not strictly meant as predicting the actual electron-to-neutral number density ratio, but is useful in comparisons with observations as an indicator of processes at play in the cometary coma.
\end{abstract}

Key words: comets: individual (67P/Churyumov-Gerasimenko) - molecular processes

\section{INTRODUCTION}

The ESA Rosetta orbiter is escorting comet $67 \mathrm{P} /$ Churyumov-Gerasimenko (hereafter 67P) through perihelion (on 2015 August 13 at a heliocentric distance $d \sim 1.25 \mathrm{AU}$ ) and beyond. The rendezvous with the comet occurred in 2014 August at $d \sim$ 3.6 AU, and at the end of the nominal mission on 2015 December 31, 67P will be at $d \sim 1.9 \mathrm{AU}$. The mission plan gives the unprecedented opportunity to study in detail how, for example, the activity, the chemical composition of the coma, and the interplay with the solar wind change with time and space.

Comet 67P consists of two differently sized lobes connected by a "neck region." It has an effective radius of $\sim 1.72 \mathrm{~km}$ and a rotation period of $\sim 12.4 \mathrm{hr}$ (Sierks et al. 2015). An early highlight of the escort phase of the mission was the discovery of a high D-to-H ratio of $(5.3 \pm 0.7) \times 10^{-4}$ (Altwegg et al. 2015), roughly three times higher than the Vienna Standard Mean Ocean Water value, showing that Earth's water may not predominantly have been delivered by Jupiter-family comets. As for the interaction of the coma with the solar wind, cometary pickup ions were observed early in the escort phase, and evidences of charge transfer from solar wind particles (e.g., $\mathrm{He}^{2+}+$ neutral $\rightarrow \mathrm{He}^{+}+$ion) to cometary neutrals have been provided (Goldstein et al. 2015; Nilsson et al. 2015). The cometary coma has hitherto been too dilute to effectively cool the electron population. As a consequence, high electron temperatures, of $\sim 3-10 \mathrm{eV}$, have been observed from the start of the escort phase until present (2015 May; A. I. Eriksson et al. 2015, in preparation; M. Galand et al. 2015, in preparation).

In the present modeling study we are concerned with the electron-to-neutral number density ratio, $n_{\mathrm{e}} / n_{\mathrm{N}}$, in the cometary coma of 67P and how it varies with cometocentric and heliocentric distance. We shall assume that photoionization is the principal source of ion-electron pair formation in the coma of 67P. In optically thin media, such as in the comae of weakly outgassing comets, it is for many purposes sufficient for modelers to adopt photoionization frequencies rather than to treat in detail the attenuation of the impinging solar EUV radiation through the Beer-Lambert law. This will likely be the case for most of the Rosetta mission and possibly even at perihelion, where significant attenuation, at least in the subsolar direction, is only predicted for cometocentric distances less than $\sim 10 \mathrm{~km}$ (Vigren \& Galand 2013; see also Section 4.6). In Section 2 we compute partial photoionization frequencies for $\mathrm{H}_{2} \mathrm{O}, \mathrm{CO}$, and $\mathrm{CO}_{2}$ (the dominant molecules in the coma of 67P; Hässig et al. 2015) at $1 \mathrm{AU}$ using the cross-section sets of Schunk \& Nagy (2009) and solar EUV spectra associated with a few selected dates. We then focus particularly on the total photoionization frequency of $\mathrm{H}_{2} \mathrm{O}$ during the pre-perihelion escort phase.

The varying photoionization frequency of $\mathrm{H}_{2} \mathrm{O}$ is used together with an adopted radial speed of the cometary neutrals to generate a simple analytical expression for the $n_{\mathrm{e}} / n_{\mathrm{N}}$ ratio, $G_{\mathrm{e} / \mathrm{N}}(r, d)$ as a function of cometocentric distance, $r$, and heliocentric distance, $d$. The expression is derived with the assumptions of a field-free and chemistry-free radially expanding coma (FFCF model). We present in Section 3 a closer description of the FFCF model and a parameterization of $G_{\mathrm{e} / \mathrm{N}}(r, d)$. The benefit of focusing on $n_{\mathrm{e}} / n_{\mathrm{N}}$ instead of simply $n_{\mathrm{e}}$ is that it, to a large extent, removes the need to account for the variability in space and time of the neutral outgassing from the cometary nucleus. The underlying assumption of a radially expanding neutral background, dropping in number density as $r^{-2}$ (for fixed latitude and longitude; see Section 3), is indeed confirmed by measurements around the comet in the early escort phase (Hässig et al. 2015). As such, comparisons between FFCF derived $n_{\mathrm{e}} / n_{\mathrm{N}}$ ratios and observations can reveal phenomena beyond the variability in the neutral outgassing. In Section 4 we review/discuss (some of) the effects/processes that can cause deviations between modelderived and observed (or actual) $n_{\mathrm{e}} / n_{\mathrm{N}}$ ratios. As examples, an observed ratio significantly higher than the FFCF value can serve as an indication of the importance of additional ionization 
sources, while an observed ratio markedly lower than the FFCF value can result, for example, from ion acceleration by the presence of electric fields or, near perihelion, by the increased importance of dissociative recombination as a plasma neutralizing mechanism. We emphasize also, in the concluding Section 5, that close agreements between values from the analytical expression and the observed $n_{\mathrm{e}} / n_{\mathrm{N}}$ ratios can be coincidental (with various effects nearly canceling out), thus not necessarily proving that the underlying assumptions in the FFCF model are strictly valid.

The $n_{\mathrm{e}} / n_{\mathrm{N}}$ ratio is an observable from Rosetta multiinstrumental in situ measurements. Measurements by the Rosetta Orbiter Spectrometer for Ion and Neutral Analysis (ROSINA; Balsiger et al. 2007) give the total number density and relative composition of the neutral part of the coma (Hässig et al. 2015) and the relative abundances of the ion population (Fuselier et al. 2015). Measurements by the dual Langmuir probe (LAP; Eriksson et al. 2007) give, for example, total electron (and ion) number densities (Edberg et al. 2015), as well as the electron temperature. Electron number densities are also accessible through measurements by the Mutual Impedance Probe (MIP; Trotignon et al. 2007; Edberg et al. 2015). The $n_{\mathrm{e}} / n_{\mathrm{N}}$ ratio has hitherto been observationally derived within reasonable error margins only for 2014 mid-October (see Edberg et al. 2015 and Section 3), where LAP-derived ion number densities could be compared with MIP-derived $n_{\mathrm{e}}$ and found to be in good agreement. Maps of $n_{\mathrm{e}} / n_{\mathrm{N}}$ around the comet at different heliocentric distances, $d$, are, however, anticipated in the near future following (cross-)instrument calibrations. It is stressed that the main purpose with our analytical expression of $n_{\mathrm{e}} / n_{\mathrm{N}}$ is that future comparisons with observationally derived ratios can provide a quick indicator of processes at play in the cometary coma.

\section{PHOTOIONIZATION FREQUENCIES}

\subsection{Partial Photoionization Frequencies of $\mathrm{H}_{2} \mathrm{O}, \mathrm{CO}_{2}$, and $\mathrm{CO}$}

Three solar spectra from measurements by the Thermosphere Ionosphere Mesosphere Energy and Dynamics/Solar EUV Experiment (TIMED/SEE; Level 3, with a resolution of $1 \mathrm{~nm}$; see Woods et al. 2005) are used to compute and compare the partial photoionization frequencies of $\mathrm{H}_{2} \mathrm{O}, \mathrm{CO}_{2}$, and $\mathrm{CO}$. We have selected 2014 August 1 and 2015 March 1. For perihelion we have used the solar EUV spectrum from 2005 January 2 $\left(F_{10.7}=100 \times 10^{-22} \mathrm{Wm}^{-2} \mathrm{~Hz}^{-1}\right)$, which is expected to represent conditions to be encountered in 2015 August (Vigren \& Galand 2013).

All partial photoionization cross sections are taken from Schunk \& Nagy (2009). The frequency for a photoionization process of a neutral molecule $A$ yielding the production of an ion $B$ is retrieved from

$$
f_{A \rightarrow B}=\int I(\lambda) \sigma_{A \rightarrow B}(\lambda) d \lambda,
$$

where $\lambda$ is the wavelength of solar EUV radiation, $I$ is the spectral intensity of the solar flux, and $\sigma$ is the cross section for the process. The integral is calculated from $0.5 \mathrm{~nm}$ to the threshold wavelength.

The derived partial photoionization frequencies for $\mathrm{H}_{2} \mathrm{O}$, $\mathrm{CO}$, and $\mathrm{CO}_{2}$ at $1 \mathrm{AU}$ are shown in Table 1 . Included in the table, for qualitative comparisons, are the corresponding frequencies at solar quiet and active conditions as presented in Huebner et al. (1992) (and later also in Huebner \& Mukherjee 2015). Their solar flux model for quiet conditions was in particular (for relevant wavelengths down to $27 \mathrm{~nm}$ ) based on measurements by the extreme-ultraviolet spectrometer aboard the Orbiting Solar Observatory 3 (Hall \& Hinteregger 1970), and for the active Sun spectral irradiances were shifted according to the Atmospheric Explorer E data of Hinteregger (1981) as presented by Lean (1987). For the major ionization channels $\left(\mathrm{H}_{2} \mathrm{O}^{+}\right.$from $\mathrm{H}_{2} \mathrm{O}, \mathrm{CO}^{+}$from $\mathrm{CO}, \mathrm{CO}_{2}^{+}$from $\left.\mathrm{CO}_{2}\right)$ the present results are intermediate of the frequencies given in Huebner et al. (1992) for solar quiet and active conditions. In addition, for all three molecules, our ranking of product ions is similar to Huebner et al. (1992). It is noteworthy that there is a reduction in the ratio of $\mathrm{CO}_{2} / \mathrm{H}_{2} \mathrm{O}$ ionization frequencies, from values of 2.0-2.2 (Huebner et al. 1992) to values of $~ 1.6$. As for the relative yields of product ions the most prominent differences with respect to the work of Huebner et al. (1992) are (1) the somewhat decreased yield of $\mathrm{H}_{2} \mathrm{O}^{+}$production and increased yield of $\mathrm{H}^{+}$production in the photoionization of $\mathrm{H}_{2} \mathrm{O}$ and (2) the decreased yield of $\mathrm{O}^{+}$and $\mathrm{C}^{+}$production in the photoionization of $\mathrm{CO}$. The relative yields of product ions in the photoionization of $\mathrm{CO}_{2}$ are in good agreement with values from Huebner et al. (1992).

\subsection{Total Photoionization Frequency of $\mathrm{H}_{2} \mathrm{O}$ during the Pre-perihelion Phase}

In Figure 1(a) we show the heliocentric distance of $67 \mathrm{P}$ against the number of days passed since 2014 July 31 . Figure 1(b) shows the total photoionization frequency of $\mathrm{H}_{2} \mathrm{O}$ at $1 \mathrm{AU}$ as calculated on a daily basis from the TIMED/SEE solar EUV flux data sets and the cross-section set of Schunk \& Nagy (2009). Included in the figure is also a linear fit of the full data set, and this displays a weakly descending trend. Combining the linear fit with the heliocentric distance time dependence gives the following function of the photoionization frequency as a function of $d$ (in $\mathrm{AU}$ ):

$$
f_{\mathrm{H}_{2} \mathrm{O}}(d)=(0.21 d+7.03) \times 10^{-7} / d^{2} .
$$

The division by $d^{2}$ accounts for the fact that the photoionization frequency is inversely proportional to the square of the heliocentric distance.

Clearly, over the investigated time period the variation in the daily averaged solar EUV spectra is dominated by the variation with the Sun's $\sim 27$ day rotation period. Using the fit function gives, however, a value that is within $17 \%$ (and for most days within $10 \%$ ) of the computed daily photoionization frequency. If Equation (2) is extrapolated to perihelion ( $d=1.25 \mathrm{AU})$, the associated photoionization frequency (normalized to $1 \mathrm{AU}$ ) of $\mathrm{H}_{2} \mathrm{O}\left(7.29 \times 10^{-7} \mathrm{~s}^{-1}\right)$ is $\sim 16 \%$ higher than the "perihelion prediction" provided in Table 1 . It is noted that TIMED is an Earth-based satellite and that there is a phase difference between the Earth and 67P that varies with time (e.g., the phase shifts on 2014 September 9, 2014 October 17, 2014 December 8 , and 2015 January 29 were $\sim 53^{\circ}, 85^{\circ}, 128^{\circ}$, and $171^{\circ}$, respectively, corresponding to $\sim+4$ days, +5 days, +9 days, and +12 days, respectively). To better pinpoint the daily averaged $\mathrm{H}_{2} \mathrm{O}$ photoionization frequency, this phase shift should be taken into account. We have here tested shifting the spectra by $1,2, \ldots, 13$ days and found that Equation (2) still is accurate within $20 \%$. 
Table 1

Calculated Ionization Frequencies, $f$ (in s ${ }^{-1}$ ), at $1 \mathrm{AU}$ with Comparisons to Values from Huebner et al. (1992) as Derived for Solar Minimum and Maximum Conditions

\begin{tabular}{|c|c|c|c|c|c|c|}
\hline Molecule & Ion & $f_{\text {perihelion }}$ & $f_{15, \text { Mar } 1}$ & $f_{14, \text { Aug } 1}$ & $f_{\mathrm{H} 92, \text { quite }}$ & $f_{\mathrm{H} 92, \text { active }}$ \\
\hline \multirow[t]{4}{*}{$\overline{\mathrm{H}_{2} \mathrm{O}}$} & $\mathrm{H}_{2} \mathrm{O}^{+}$ & $4.68(-7)^{\mathrm{a}}$ & $5.33(-7)$ & $6.00(-7)$ & $3.31(-7)$ & $8.28(-7)$ \\
\hline & $\mathrm{OH}^{+}$ & $1.09(-7)$ & $1.49(-7)$ & $1.64(-7)$ & $5.54(-8)$ & $1.51(-7)$ \\
\hline & $\mathrm{H}^{+}$ & $4.48(-8)$ & $6.68(-8)$ & $7.19(-8)$ & $1.31(-8)$ & $4.07(-8)$ \\
\hline & Sum & $6.27(-7)$ & $7.58(-7)$ & $8.45(-7)$ & $4.05(-7)$ & $1.04(-6)$ \\
\hline \multirow[t]{2}{*}{$\mathrm{CO}$} & $\mathrm{CO}^{+}$ & $5.65(-7)$ & $7.07(-7)$ & $7.85(-7)$ & $3.80(-7)$ & $9.59(-7)$ \\
\hline & $\mathrm{C}^{+}$ & $3.28(-8)$ & $5.16(-8)$ & $5.52(-8)$ & $2.94(-8)$ & $9.88(-8)$ \\
\hline \multirow[t]{5}{*}{$\mathrm{CO}_{2}$} & $\mathrm{CO}_{2}^{+}$ & $7.94(-7)$ & $9.22(-7)$ & $1.04(-6)$ & $6.55(-7)$ & $1.76(-6)$ \\
\hline & $\mathrm{O}^{+}$ & $8.65(-8)$ & $1.35(-7)$ & $1.44(-7)$ & $6.38(-8)$ & $2.11(-7)$ \\
\hline & $\mathrm{CO}^{+}$ & $5.79(-8)$ & $9.16(-8)$ & $9.74(-8)$ & $5.02(-8)$ & $1.66(-7)$ \\
\hline & $\mathrm{C}^{+}$ & $3.91(-8)$ & $6.34(-8)$ & $6.70(-8)$ & $2.89(-8)$ & $1.07(-7)$ \\
\hline & Sum & $9.78(-7)$ & $1.21(-6)$ & $1.35(-6)$ & $7.98(-7)$ & $2.24(-6)$ \\
\hline
\end{tabular}

Note.

a $4.68(-7)$ should be read as $4.68 \times 10^{-7}$.
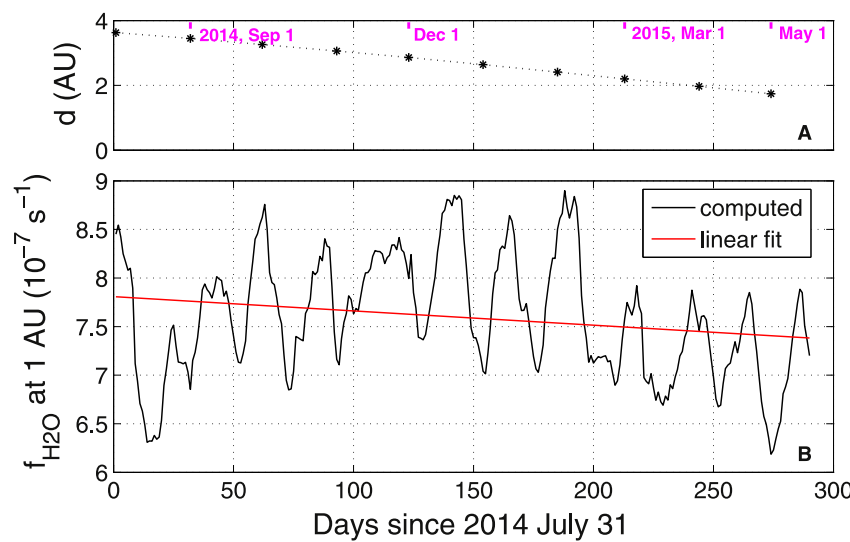

Figure 1. Shown against the day number since 2014 July 31 (day 0) is (a) the heliocentric distance of 67P and (b) the computed photoionization frequency of $\mathrm{H}_{2} \mathrm{O}$ at $1 \mathrm{AU}$ and the associated best linear fit. As for the total photoionization frequencies of $\mathrm{CO}_{2}$ and $\mathrm{CO}$ at $1 \mathrm{AU}$ (not shown), we recommend multiplying the displayed $\mathrm{H}_{2} \mathrm{O}$ photoionization frequencies by 1.6 and 1.1 , respectively. The resulting frequencies are estimated accurate to within $10 \%$.

\section{ANALYTIC EXPRESSION OF ELECTRON-TO- NEUTRAL NUMBER DENSITY RATIO}

An analytic expression (Equation (4) below) of the electronto-neutral number density ratio $G_{\mathrm{e} / \mathrm{N}}(r, d)$ at the cometocentric distance $r$ and the heliocentric distance $d$ is derived from a series of assumptions and approximations.

i. Neutrals travel radially outward with a constant speed $u_{\mathrm{N}}$ independent of $r$ and $d$. The neutral number density is given by $Q /\left(4 \pi r^{2} u_{\mathrm{N}}\right)$, with $Q$ the molecular outgassing rate from the nucleus (see, e.g., Haser 1957). From Haser's (1957) expression of the neutral number density we have omitted the exponential factor accounting for the photodissociation and photoionization of neutrals. For heliocentric distances $>1.25 \mathrm{AU}$ and with expansion speeds of $>0.5 \mathrm{~km} \mathrm{~s}^{-1}$ (see below) the photodissociation and/or photoionization scale length of a cometary molecule (e.g., $\mathrm{H}_{2} \mathrm{O}$ ) exceeds $10^{4} \mathrm{~km}$, which is at least two orders of magnitude greater than the cometocentric distances we focus on here. ii. Major primary ions produced by photoionization travel radially outward with the same speed as the neutrals.

iii. Photoionization is the only considered ionization source, and attenuation of the EUV irradiation is negligible. Photoionization processes yielding more than one free electron are neglected (minor correction, not further discussed).

iv. Grain charging and plasma neutralization through dissociative recombination are neglected.

v. Ions and electrons from the solar wind are neglected.

Under these conditions the electron number density at $r$ and $d$ can be calculated by integrating electron production from $r_{\mathrm{C}}$ (the cometary radius) to $r$ and dividing the thereby derived electron number flux by the mantle area at $r$ and the radial (drift) velocity:

$$
n_{\mathrm{e}}(r, d)=\frac{1}{4 \pi u_{\mathrm{N}} r^{2}} \sum_{j} \int_{r_{0}=r_{\mathrm{C}}}^{r} f_{j} g_{j} \frac{Q}{4 \pi u_{\mathrm{N}} r_{0}^{2}} 4 \pi r_{0}^{2} d r_{0},
$$

where $f_{j}$ and $g_{j}$ denote the ( $d$-dependent) ionization frequency (at the comet location) and the relative abundance of the neutral species $j$, respectively. Performing the integration and dividing by the neutral number density expression (see (i) above), one obtains

$$
\frac{n_{\mathrm{e}}}{n_{\mathrm{N}}}(r, d)=\frac{\left(r-r_{c}\right)}{u_{\mathrm{N}}} \sum_{j} f_{j}(d) g_{j} .
$$

The underlying model to calculate the $n_{\mathrm{e}} / n_{\mathrm{N}}$ ratio is here referred to as the FFCF model.

In the following we consider for simplicity a pure $\mathrm{H}_{2} \mathrm{O}$ cometary coma. This gives, using Equation (2), setting the comet radius to $2 \mathrm{~km}$, and using $u_{\mathrm{N}}=650 \mathrm{~m} \mathrm{~s}^{-1}$ as adopted from measurements by the Microwave Instrument for the Rosetta Orbiter (MIRO; Gulkis et al. 2015),

$$
G_{\mathrm{e} / \mathrm{N}}(r, d)=(0.32 d+10.82) \times 10^{-7} \times(r-2) / d^{2},
$$

with $r$ inserted in $\mathrm{km}$ and $d$ inserted in AU. Figure 2 illustrates for a selection of heliocentric distances how $G_{\mathrm{e} / \mathrm{N}}$ vary with cometocentric distance $r<50 \mathrm{~km}$. 


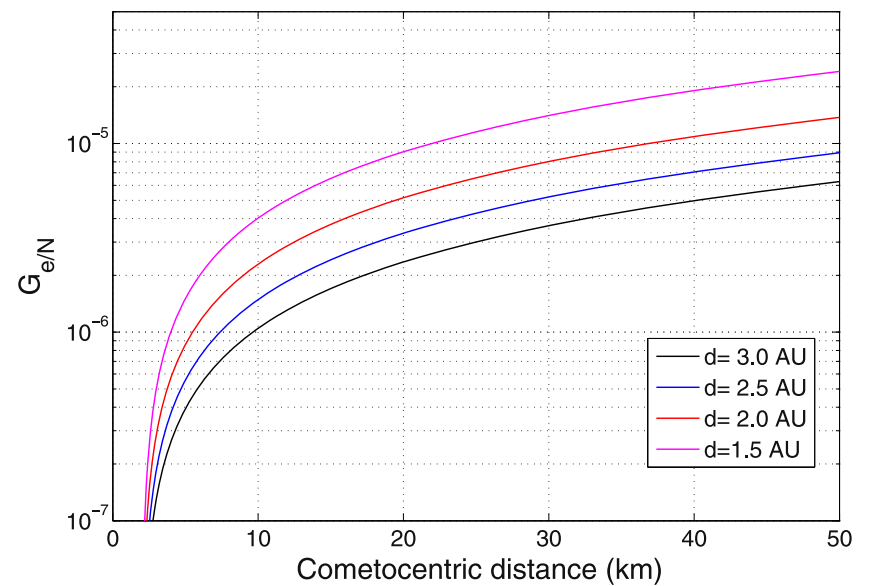

Figure 2. Guiding electron-to-neutral number density ratio (Equation (4)) as a function of cometocentric distance for a selection of heliocentric distances.

Edberg et al. (2015) combined LAP, MIP, and ROSINA/ COPS (comet pressure sensor) measurements in 2014 midOctober, showing that the electron-to-neutral number density ratio at $d \sim 3.1 \mathrm{AU}$ and $r \sim 10 \mathrm{~km}$ varied within a range of $(1-2) \times 10^{-6}$. This is in accord with the $G_{\mathrm{e} / \mathrm{N}}$ value of $9.83 \times 10^{-7}$ computed at the same $r$ and $d$. Despite this particular resemblance, $G_{\mathrm{e} / \mathrm{N}}$ is not to be viewed as a prediction of the actual electron-to-neutral number density ratio (as mentioned in Section 1). Rather, it is useful in comparisons with observationally derived ratios to give insights into processes at play in the cometary coma.

\section{EFFECTS YIELDING OBSERVATIONAL DEVIATIONS FROM $G_{\mathrm{e} / \mathrm{N}}(r, d)$}

There are various effects/processes that can act to increase or decrease the actual electron-to-neutral number density ratios from the $G_{\mathrm{e} / \mathrm{N}}$ value. Here we go through some of these and discuss their separate influences on the calculated electron-toneutral number density ratio (i.e., all other parameters, assumptions, and approximations used to derive $G_{\mathrm{e} / \mathrm{N}}$ remain fixed).

\subsection{Varying Neutral Composition}

The $G_{\mathrm{e} / \mathrm{N}}(r, d)$ function was derived for a pure $\mathrm{H}_{2} \mathrm{O}$ coma, and in FFCF models the $n_{\mathrm{e}} / n_{\mathrm{N}}$ ratio is proportional to the adopted effective ionization frequency. The photoionization frequency of $\mathrm{CO}$ is comparable with $\mathrm{H}_{2} \mathrm{O}$, but the $\mathrm{CO}_{2}$ ionization frequency is higher by $\sim 60 \%$.

\subsection{Varying Outgassing Velocity}

The $G_{\mathrm{e} / \mathrm{N}}(r, d)$ value is to be multiplied by a factor $650 / u_{\mathrm{N}, a}$, with $u_{\mathrm{N}, a}$ being the actual radial speed of the neutrals (in $\mathrm{m} \mathrm{s}^{-1}$ ). For example, if the actual radial speed of the neutrals is $400 \mathrm{~m} \mathrm{~s}^{-1}$, the $G_{\mathrm{e} / \mathrm{N}}(r, d)$ should be increased by $75 \%$, and if the actual speed is $1 \mathrm{~km} \mathrm{~s}^{-1}, G_{\mathrm{e} / \mathrm{N}}(r, d)$ should be decreased by $30 \%$. There can also be radial speed gradients present with the neutrals requiring a certain radial movement before reaching terminal velocities. However, even near perihelion, where this effect is expected to be most pronounced, terminal speeds are predicted to be almost reached already by $r \sim 10 \mathrm{~km}$ (Tenishev et al. 2008).

\subsection{Varying Solar EUV Spectra}

The solar EUV intensity can vary on short timescales, and for case studies it is recommended to use a solar EUV spectrum for the day of interest corrected for the phase separation between Earth and 67P. Note, however, that the computed $\mathrm{H}_{2} \mathrm{O}$ ionization frequency at $1 \mathrm{AU}$ on 2014 August 1 is only $~ 35 \%$ higher than the corresponding perihelion prediction, and so this effect alone would under "normal" conditions not be expected to cause pronounced deviations from the $G_{\mathrm{e} / \mathrm{N}}$ ratios. Enhanced ionization frequencies can, on the other hand, result from solar flares and associated coronal mass ejection. The effect of attenuation of the incident solar irradiation is discussed in Section 4.6.

\subsection{Additional Ionization Sources}

Cravens et al. (1987) utilized electron spectra measured near comet Halley and Giacobini-Zinner (by instruments on the VEGA and International Cometary Explorer spacecraft, respectively) to determine electron impact ionization frequencies. It was shown that in the magnetosheaths of the comets, electron impact ionization is comparable with photoionization of $\mathrm{H}_{2} \mathrm{O}$. In fact, the authors addressed the possibility that the electron impact ionization frequency is a few times higher than the photoionization frequency inward of $\sim 10^{4} \mathrm{~km}$ in the coma of Halley. The authors stressed, however, that a peak of highenergy electrons (in the VEGA electron spectrum) of unknown origin was the main contributor to the high ionization frequencies. As for the coma of 67P, electron-impact ionization frequencies (of, e.g., $\mathrm{H}_{2} \mathrm{O}, \mathrm{CO}_{2}$, and $\mathrm{CO}$, for which electron impact ionization cross sections are available) can in principle be derived from the electron intensity spectra acquired by the Ion and Electron Sensor (IES; Burch et al. 2007), corrected for the spacecraft potential as determined from LAP.

An obvious source of high-energy electrons are the photoelectrons themselves. These can be energetic enough to cause further ionizations, and the thereby-produced secondary electrons can cause further ionizations, and so on. Vigren \& Galand (2013) calculated for 67P, near perihelion, the primary efficiencies-defined as the ratio between electron impact ionization rates (solar wind electrons excluded) and photoionization rates-as a function of cometocentric distance. In the optically thin domain the primary efficiency was less than $20 \%$, in agreement with model estimates from Halley at large cometocentric distances (Körözmezey et al. 1987). The predicted value increased toward the nucleus but exceeded $30 \%$ only at cometocentric distances less than $20 \mathrm{~km}$. It is interesting to note that for comets being significantly $(\sim 3$ orders of magnitude) more active than $67 \mathrm{P}$ at perihelion, calculated photoionization rate profiles exhibit a double structure, and near the lower photoionization peak the contribution of electron-impact ionization to the total ionization rate exceeds the contribution from photoionization by roughly an order of magnitude (Bhardwaj 2003).

Cravens et al. (1987) stated also that the $\mathrm{H}_{2} \mathrm{O}^{+}$production rate from charge exchange of cometary $\mathrm{H}_{2} \mathrm{O}$ with solar wind protons is comparable with the photoionization frequency, at least during solar minimum conditions. This has also been highlighted subsequently (e.g., Khabibrakhmanov \& Summers 1997) and is furthermore supported by combining the solar wind parameters by Hansen et al. (2007) with more recent experimental and theoretical results for the charge transfer 

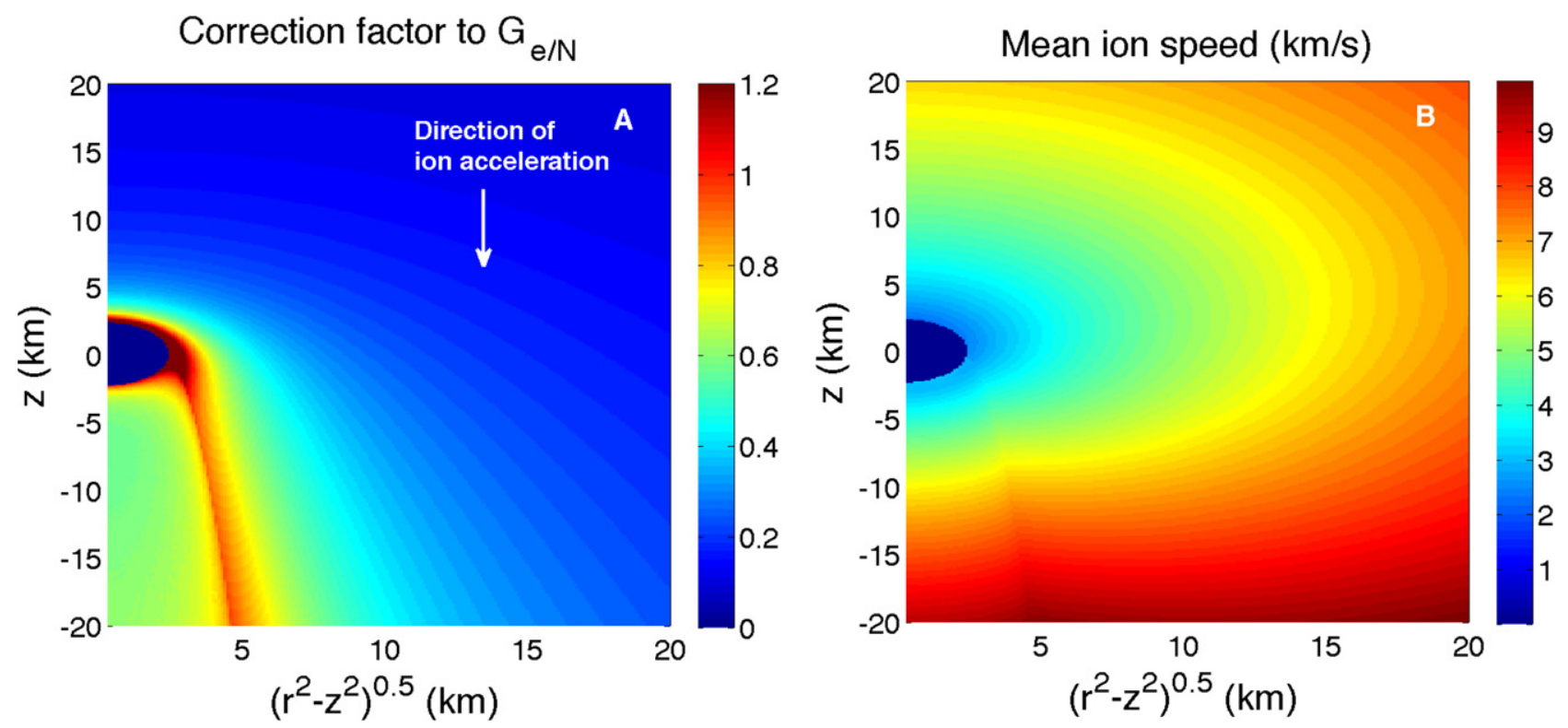

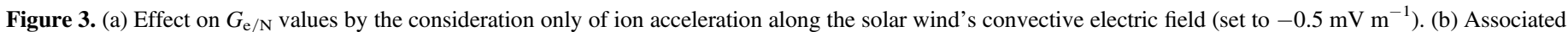

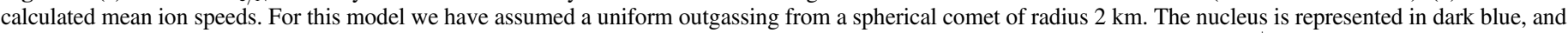

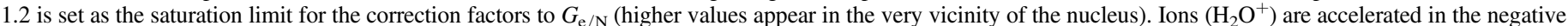

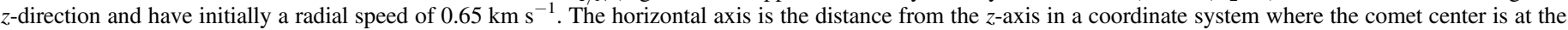
origin. The tail in (a) is not pointing in the anti-sunward direction, but nearly perpendicular to it.

cross section as a function of proton energy (Lindsay et al. 1997; Mada et al. 2007). In addition, as mentioned in Section 1, observations by the ICA and IES instruments have shown charge transfer occurring in the coma of 67P (Goldstein et al. 2015; Nilsson et al. 2015). The charge transfer process does not represent a direct source of free electrons, although possibly indirectly through the buildup of electric fields preserving charge neutrality. We will not investigate this effect further in the present study. We note, however, that the importance of charge transfer is suppressed near the nucleus by the deflection of solar wind protons in response to the generation of cometary pickup ions (see, e.g., Rubin et al. 2014). Furthermore, within the diamagnetic cavity, in case such a region forms, the coma is shielded from the solar wind.

Finally, it is merely noted that our model does not account for photoelectrons and sputtered secondary electrons from the cometary nucleus (the charging of a cometary nucleus at large heliocentric distances is discussed, e.g., by Mendis et al. 1981).

\subsection{Effect of Electric Fields}

Modeling in detail the interplay between the cometary coma, solar wind and electromagnetic field environment is complex and beyond the scope of the present paper. Readers are referred to Hansen et al. (2007), Koenders et al. (2013, 2015), Rubin et al. (2014), and references therein, for descriptions of MHD and hybrid simulations of the plasma environment of $67 \mathrm{P}$, though the focuses of those studies were not primarily on the near nucleus environment. Here we isolate, and consider separately, the effect on the ion number densities of (i) the solar wind convective electric field (Section 4.5.1) and (ii) the charge separation electric field in an isothermal (constant electron temperature) cometary plasma (Section 4.5.2). In both cases (calculations), the electric field affects the positive ions (restricted to $\mathrm{H}_{2} \mathrm{O}^{+}$), while the electron population simply preserves charge neutrality. In both cases, magnetic field effects on the electron motion are neglected, restricting the validity to low magnetic field strengths (or to directions from the nucleus close to parallel with the magnetic field). Finally, we note that the second calculation is also simplified by the consideration of a uniformly distributed plasma around the nucleus (with the ambipolar electric field pointing in the radial direction).

\subsubsection{Accounting Only for the Solar Wind Convective Electric Field}

Near the onset of cometary activity and when there is no significant mass loading of the solar wind, newborn cometary ions are accelerated in the direction of the solar wind's convective electric field, for which a reasonable magnitude at $d=3-3.5 \mathrm{AU}$ is in the range of a few tenths to a few $\mathrm{mV} \mathrm{m}^{-1}$ (e.g., Hansen et al. 2007). Figure 3(a) illustrates how $G_{\mathrm{e} / \mathrm{N}}$ values in the near nucleus environment (we consider here scales much smaller than an ion gyroradius), assuming a uniform outgassing from the comet, are affected by only accounting for ion acceleration along the convective electric field (using an electric field of $E_{z}=-0.5 \mathrm{mV} \mathrm{m}^{-1}$ and only considering water ions). Figure 3(b) shows associated mean ion speeds. The mathematics of the model is described in Appendix A. It is stressed that the model is limited to a very weakly outgassing comet. In fact, ICA and IES observed deflection of the solar wind early (even beyond $3 \mathrm{AU}$ ) in the escort phase (Goldstein et al. 2015; Nilsson et al. 2015).

The asymmetric correction factors in Figure 3(a) correspond to an asymmetric plasma distribution around the comet. The tail is not pointing in the anti-sunward direction but in the direction of the convective electric field. A similar structure, although with lower spatial resolution, appears also from hybrid simulations of $67 \mathrm{P}$ at large heliocentric distances, prior to significant mass loading (e.g., Hansen et al. 2007). 
Table 2

$\mathrm{H}_{2} \mathrm{O}$ Total Ionization Frequencies Relative to Unattenuated Value for Combinations of Outgassing Rate, $Q$, Cometocentric Distance, $r$, and Solar Zenith Angle, SZA

\begin{tabular}{lccccccccc}
\hline \hline & \multicolumn{4}{c}{$Q=1 \times 10^{27} \mathrm{~s}^{-1}$} & & \multicolumn{4}{c}{$Q=1 \times 10^{28} \mathrm{~s}^{-1}$} \\
\cline { 2 - 7 } \cline { 7 - 9 }$r \backslash \mathrm{SZA}$ & $0^{\circ}$ & $30^{\circ}$ & $60^{\circ}$ & $80^{\circ}$ & & $0^{\circ}$ & $30^{\circ}$ & $60^{\circ}$ & $80^{\circ}$ \\
\hline $5 \mathrm{~km}$ & 0.97 & 0.97 & 0.96 & 0.95 & & 0.73 & 0.71 & 0.67 & 0.62 \\
$10 \mathrm{~km}$ & 0.98 & 0.98 & 0.98 & 0.98 & & 0.85 & 0.84 & 0.82 & 0.79 \\
$30 \mathrm{~km}$ & 0.99 & 0.99 & 0.99 & 0.99 & & 0.95 & 0.95 & 0.94 & 0.93 \\
$50 \mathrm{~km}$ & 1.00 & 1.00 & 1.00 & 1.00 & & 0.97 & 0.97 & 0.96 & 0.96 \\
$100 \mathrm{~km}$ & 1.00 & 1.00 & 1.00 & 1.00 & & 0.99 & 0.98 & 0.98 & 0.98 \\
\hline
\end{tabular}

\subsubsection{Accounting Only for a Charge Separation Electric Field}

Treating the comet as a pure $\mathrm{H}_{2} \mathrm{O}$ outgassing point source reduces the FFCF-derived electron-to-neutral number density ratio to the expression

$$
n_{\mathrm{e}}^{*} / n_{\mathrm{N}}=\frac{r f_{\mathrm{H}_{2} \mathrm{O}}}{u_{\mathrm{N}}} .
$$

Equation (5), where the asterisk is present to indicate that the ratio is derived through the point-source treatment, is a reasonable approximation of the FFCF-derived $n_{\mathrm{e}} / n_{\mathrm{N}}$ ratio for cometocentric distances larger than a few cometary radii. As the neutral number density drops as $r^{-2}$, the electron number density drops as $1 / r$. Neglecting the solar wind's convective electric field (and ion-neutral momentum exchange) and assuming further that the electron temperature is constant (at a high value of $T_{\mathrm{e}} \sim 10^{5} \mathrm{~K}$ roughly corresponding to $10 \mathrm{eV}$ ), this gradient in electron number density sets up an outwardpointing ambipolar electric field, $E_{r}$, that (by neglecting the motional electric field) is inversely proportional to $r$. The relation is given in Appendix B (Equation (14)). We show there also that the presence of such a field (and such a field only) in an isothermal coma is consistent with (results in) an electron number density proportional to $1 / r$. There is, however, a reduction in the $n_{\mathrm{e}} / n_{\mathrm{N}}$ ratio (as given by the right-hand side of Equation (5)), approximately by the multiplicative factor $\sqrt{\pi \eta}-2 \eta$, where the dimensionless parameter

$$
\eta=\frac{m_{\mathrm{I}} u_{\mathrm{N}}^{2}}{2 k_{B} T_{\mathrm{e}}},
$$

with $m_{\mathrm{I}}$ the ion mass. Note that the approximation only holds for combinations of high $T_{\mathrm{e}}$ and low to moderate $u_{\mathrm{N}}$ giving $\eta \ll 1$. As an example, with $u_{\mathrm{N}}=650 \mathrm{~m} \mathrm{~s}^{-1}, m_{\mathrm{I}}=18 \mathrm{amu}$, and with an electron temperature of $10^{5} \mathrm{~K}$, one obtains $\eta \sim 0.0046$ and consequently $\sqrt{\pi \eta}-2 \eta \approx 0.1$.

\subsection{Attenuation of the EUV Flux}

Near perihelion, attenuation of the impinging solar EUV flux (in the column toward the Sun) can reduce the ambient photoionization frequencies. In Table 2 we show for two different molecular outgassing rates, $Q$, and for four different incident irradiation angles how solar EUV photoabsorption affects the total $\mathrm{H}_{2} \mathrm{O}$ ionization frequency. The values have been derived from the Beer-Lambert law using the 2015 March 1 TIMED/SEE solar EUV spectrum with absorption and total ionization cross sections from Schunk \& Nagy (2009) and assuming a spherically symmetric coma with a Haser (1957) type $\mathrm{H}_{2} \mathrm{O}$ background (using a radial speed of $0.65 \mathrm{~km} \mathrm{~s}^{-1}$ and ignoring decay in neutral densities due to photoprocesses). The higher of the $Q$ values $\left(1 \times 10^{28} \mathrm{~s}^{-1}\right)$ is in the vicinity of predictions near perihelion (see, e.g., Snodgrass et al. 2013 and references therein), and the attenuation effect is considerable (ionization frequencies decreased by $>20 \%$ ) only inward of $\sim 10 \mathrm{~km}$ even near the terminator.

\subsection{Plasma Loss through Dissociative Recombination}

Near perihelion, when the $\mathrm{H}_{2} \mathrm{O}$ number densities become high enough, $\mathrm{H}_{2} \mathrm{O}$ molecules can effectively cool the electron population, possibly down to a few hundred or even a few tens of $\mathrm{K}$ (depending on the neutral temperature). This electron temperature lowering, combined with the high plasma number densities, makes dissociative recombination an important plasma neutralizing mechanism.

To give an idea of the influence, we notice that the $n_{\mathrm{e}} / n_{\mathrm{N}}$ ratios from the perihelion simulation by Vigren \& Galand (2013, for which the solar flux undergoes a weak attenuation, the solar zenith angle is set to $0^{\circ}$, and $T_{\mathrm{e}}$ varies with $r$ from $\sim 150 \mathrm{~K}$ near the surface to $\sim 10 \mathrm{~K}$ at $100 \mathrm{~km}$ ) were $\sim 2.3,4.4$, 6.5 , and $17.6 \times 10^{-6}$ at cometocentric distances of $10,20,30$, and $100 \mathrm{~km}$, respectively. The corresponding $G_{\mathrm{e} / \mathrm{N}}$ values (setting $d=1.29$ as used as perihelion distance in Vigren \& Galand 2013) are factors of about 2 to $>3$ higher: $~ 5.4,12.2$, 18.9 , and $66.2 \times 10^{-6}$, respectively.

\subsection{Nanograin Charging}

In their parameter study, Vigren et al. (2015) showed that if nanograins are present at a level of $\sim 1 \%$ with respect to the mass of the gas, a significant level of electron depletion is anticipated in the innermost coma of 67P near perihelion. The study was motivated by recent Cassini observations in the water-dominated plume of the Saturnian satellite Enceladus, with surprisingly low electron-to-ion number density ratios $(<5 \%)$ that partly were attributed to nanograin charging (Morooka et al. 2011; Hill et al. 2012). Nanograin charging may potentially set $n_{\mathrm{e}} / n_{\mathrm{N}}$ ratios far below the $G_{\mathrm{e} / \mathrm{N}}$ value in the coma of $67 \mathrm{P}$ in its most active stages. However, if the guiding value instead is used for the ion-to-neutral number density ratio, dramatic deviations from observations are not expected. This is because it is mainly the electrons that charge the grains; the depletion of gas-phase positive ions is slower as a result of their lower thermal velocities (see Vigren et al. 2015).

\section{SUMMARY}

We have computed $1 \mathrm{AU}$ partial photoionization frequencies of $\mathrm{H}_{2} \mathrm{O}, \mathrm{CO}_{2}$, and $\mathrm{CO}$ at different stages of the ESA Rosetta mission. For major products the results are, as expected, intermediate of values presented at solar quiet and active conditions by Huebner et al. (1992). We have investigated how the total photoionization frequency of $\mathrm{H}_{2} \mathrm{O}$ at $1 \mathrm{AU}$ varies in time from 2014 August 1 to 2015 May 20 and presented a linear fit of this frequency versus the heliocentric distance (see Equation (2)). An FFCF model calculation assuming outward radial transport, similar speeds of neutrals and ions, no plasma neutralization through dissociative recombination, and no grain charging yielded Equation (4) as a simple analytical expression for the electron-to-neutral number density ratio, $G_{\mathrm{e} / \mathrm{N}}(r, d)$, at cometocentric distance $r$ and heliocentric distance $d$. For 
$r=10 \mathrm{~km}$ and $d=3.1$ AU the observationally derived $n_{\mathrm{e}} / n_{\mathrm{N}}$ ratios of $(1-2) \times 10^{-6}$ (Edberg et al. 2015) match the $G_{\mathrm{e} / \mathrm{N}}$ value of $9.83 \times 10^{-7}$.

The $G_{\mathrm{e} / \mathrm{N}}$ function is not to be viewed as a predictor of the actual $n_{\mathrm{e}} / n_{\mathrm{N}}$ ratio. It is rather of use in comparisons with observations as an indicator of processes affecting the ionization balance. Resemblance with observations is not, on its own, proving that the underlying assumptions of the simplified FFCF model by necessity are valid, as multiple effects may be canceling out. We have discussed in Section 4 some of the effects that can cause deviations between the actual $n_{\mathrm{e}} / n_{\mathrm{N}}$ ratios and those given by Equation (4). For example, near perihelion, when the inner ionosphere is expected to be more chemically controlled, the $G_{\mathrm{e} / \mathrm{N}}$ values are likely to give too high values as the FFCF model does not account for plasma loss through dissociative recombination. At earlier stages the $G_{\mathrm{e} / \mathrm{N}}$ values may overshoot observed ratios owing to the presence of electric fields (of different origins) accelerating the cometary ion population to speeds of several to tens of $\mathrm{km} \mathrm{s}^{-1}$. The potential importance of other ionization/ion sources than photoionization has been discussed. For example, at early stages, prior to significant solar wind deflection, the production of $\mathrm{H}_{2} \mathrm{O}^{+}$from charge transfer between solar wind protons and cometary $\mathrm{H}_{2} \mathrm{O}$ can be significant. As for electron impact ionization, it should be possible to estimate its contribution to the total ionization rate from IES measurements of suprathermal electron intensities corrected for the spacecraft potential as measured by LAP.

In order to provide FFCF model-derived $n_{\mathrm{e}} / n_{\mathrm{N}}$ ratios that are more suitable for comparisons with observation than Equation (4), the input of the model can be updated based on the daily estimates of the solar EUV flux at the comet and based on in situ measurements of the coma composition and the neutral outgassing speed (M. Galand et al. 2015, in preparation).

Research at the Swedish Institute of Space Physics is funded by the Swedish National Space Board and by the Swedish Research Council. M.G. and S.S. are partially funded by the Science \& Technology Facilities Council (STFC) through grant ST/K001051/1 to Imperial College London. We thank Hans Rullgård and Christoph Koenders for valuable input. We also thank Tom Woods and his team for providing us with TIMED/ SEE data. Finally, we thank an anonymous reviewer for constructive and useful comments.

\section{APPENDIX A \\ ION NUMBER DENSITY DISTRIBUTION IN THE PRESENCE OF THE SOLAR WIND'S CONVECTIVE ELECTRIC FIELD ACCELERATING IONS IN THE NEGATIVE $z$-DIRECTION}

We consider here scales much shorter than the pickup ion gyroradius and so neglect magnetic forces. The constant acceleration along the electric field is given by $a=q E_{z} / m_{\mathrm{I}}$, where $q$ is the elementary charge and $m_{\mathrm{I}}$ is the ion mass. An ion produced at a specific location $S_{0}$ that after a time $\tau$ has coordinates $(x, y, z)$ would in the absence of the convective electric field instead have coordinates $\left(x, y, z-a \tau^{2} / 2\right)$. Note that the field initially only affects the ion motion in the $z$-direction. The ion number density at the position $(x, y, z)$ can be calculated numerically by integrating ion contributions with respect to their travel time $\tau$ :

$$
n_{\mathrm{I}}(x, y, z)=\int_{\tau=0}^{\infty} f n_{\mathrm{N}}\left(x, y, z-\frac{a \tau^{2}}{2}\right) C_{\tau} d \tau
$$

where $n_{\mathrm{N}}\left(x, y, z-a \tau^{2} / 2\right)$ is the neutral number density (see Equation (10) below) in the coordinates $\left(x, y, z-a \tau^{2} / 2\right), f$ is the ionization frequency, and the function

$$
C \tau\left(x, y, z, a, \tau, r_{\mathrm{C}}, u_{\mathrm{N}}\right)=1 \text { if } r^{\prime}-r_{c} \geqslant u_{\mathrm{N}} \tau\left(\text { else } C_{\tau}=0\right),
$$

where

$$
r^{\prime}=\sqrt{x^{2}+y^{2}+\left(z-\frac{a \tau^{2}}{2}\right)^{2}},
$$

and with $r_{\mathrm{C}}$ the cometary radius and $u_{\mathrm{N}}$ the radial speed of the water molecules. The constraint for $C_{\tau}$ is to exclude ion trajectories that would pass through the nucleus. When numerically solving the integral in Equation (7), it is typically enough to set the upper integration limit to $10 \mathrm{~s}$ (sensitivity tests are recommended). The neutral number density was, when generating Figure 3, assumed to be related to $r^{\prime}$ via a Haser (1957) like expression

$$
n_{\mathrm{N}}\left(r^{\prime}\right)=\frac{Q}{4 \pi u_{\mathrm{N}} r^{\prime 2}},
$$

where $Q$ is the molecular outgassing rate, although the method can be applied to other neutral background models as well. From a first look at Equations (7)-(9) readers may be confused by using in Equation (7) the $n_{\mathrm{N}}$ at $r^{\prime}$ (Equation (9)) rather than using the $n_{\mathrm{N}}$ at the location of the ion production. This is because account has been taken for ion density drop. Let us regard the integral as a discrete sum and focus on an individual term $\tau_{A}$ with associated $C_{\tau}=1$ and a production site $r_{A}$. Ions produced in the very vicinity of $r_{A}$ during the infinitesimally small time step $d \tau$ would in a field-free case (with $a=0 \mathrm{~m} \mathrm{~s}^{-2}$ ) contribute to an ion density at $r^{\prime}$ given by $f n_{\mathrm{N}}\left(r_{A}\right) d \tau\left(r_{A} / r^{\prime}\right)^{2}=f n_{\mathrm{N}}\left(r^{\prime}\right) d \tau$, where the rightmost factor on the left-hand side accounts for the radial expansion. In the presence of a field, acting only in the $z$-direction, the expansion correction factor is still given by $\left(r_{A} / r^{\prime}\right)^{2}$. This follows because at $\tau_{A}$ the separation (in all dimensions) of any two ions formed in the very vicinity of $r_{A}$ during the infinitesimally small time step $d \tau$ will be the same regardless of whether or not the field along $z$ is present. This is obvious for the $x$ - and $y$-dimensions (as the field along $z$ does not affect the ion motions in $x$ and $y$ in our simplified treatment) and applies also for the $z$-direction as the factor containing $a$ in the ions' kinetic equations will be exactly similar $\left(a \tau_{A}^{2} / 2\right)$.

Mean ion speeds, $u_{\mathrm{I}}$, can be approximated (neglecting the initial radial velocity $u_{\mathrm{N}}$ ) as a weighted average of $a \tau$ :

$$
u_{\mathrm{I}}(x, y, z)=\frac{\int_{\tau=0}^{\infty} a \tau f n_{\mathrm{N}}\left(x, y, z-a \tau^{2} / 2\right) C_{\tau} d \tau}{\int_{\tau=0}^{\infty} f n_{\mathrm{N}}\left(x, y, z-a \tau^{2} / 2\right) C_{\tau} d \tau} .
$$




\section{APPENDIX B \\ CHARGE SEPARATION ELECTRIC FIELD IN AN \\ ISOTHERMAL COMA: CONSISTENCY OF $n_{\mathrm{e}} \sim 1 / r$}

We consider here a coma with a neutral number density at cometocentric distance $r$ given by (e.g., Haser 1957, but omitting the exponential factor accounting for loss of neutrals through photoprocesses)

$$
n_{\mathrm{N}}(r)=\frac{Q}{4 \pi u_{\mathrm{N}} r^{2}}
$$

where $Q$ is the outgassing rate. In the point-source treatment the FFCF model gives (see Section 4.5.2)

$$
n_{\mathrm{e}, \mathrm{FFCF} *}=n_{\mathrm{I}, \mathrm{FFCF} *}=\frac{r f_{\mathrm{H}_{2} \mathrm{O}}}{u_{\mathrm{N}}} n_{\mathrm{N}}(r),
$$

where we consider a water-dominated coma and where we set the electron number density equal to the ion number density $n_{\mathrm{I}}$. The FFCF model in the point-source treatment (stressed by an asterisk) thus gives an $1 / r$ dependence in $n_{\mathrm{e}}$. With a constant $T_{\mathrm{e}}$ the ambipolar electric field pointing in the radial direction can be approximated as (e.g., Cravens et al. 1984)

$$
E_{r}=-\frac{1}{n_{\mathrm{e}} q} \frac{d p_{\mathrm{e}}}{d r}=\frac{k_{B} T_{\mathrm{e}}}{q r}
$$

where

$$
p_{\mathrm{e}}(r)=n_{\mathrm{e}}(r) k_{B} T_{\mathrm{e}}
$$

is the electron pressure. As such, ions at $r$ experience an outward acceleration given by

$$
a(r)=\frac{q E_{r}}{m_{\mathrm{I}}}=\frac{k_{B} T_{\mathrm{e}}}{m_{\mathrm{I}} r} .
$$

Consider a spherical shell at $r_{0}$ with thickness $u_{\mathrm{N}} d t$ ( $u_{\mathrm{N}}$ being the initial radial speed of the ions). The number of ions produced in the shell during $d t$ is $f n_{\mathrm{N}}\left(r_{0}\right) 4 \pi r_{0}^{2} u_{\mathrm{N}} d t$. This group of ions travel to a position $r$, by which time they will be accelerated to a speed $u_{\mathrm{I}, r}\left(r_{0}\right)$ (see below). Accordingly, conservation of this group of ions requires $d n_{\mathrm{I}, r}\left(r_{0}\right) 4 \pi r^{2} u_{\mathrm{I}, r}\left(r_{0}\right)=4 \pi r_{0}^{2} f n_{\mathrm{N}}\left(r_{0}\right) u_{\mathrm{N}} d t$, where $d n_{\mathrm{I}, r}\left(r_{0}\right)$ is the contribution to the ion density at $r$ from ions created at $r_{0}$. Noting that $u_{\mathrm{N}} d t=d r_{0}$ and that $4 \pi r_{0}^{2} u_{\mathrm{N}} n_{\mathrm{N}}\left(r_{0}\right)=Q$, solving for $d n_{\mathrm{I}, r}\left(r_{0}\right)$, and integrating from the surface of the comet to $r$ yields

$$
n_{\mathrm{I}, A F}(r)=\frac{Q f}{4 \pi u_{\mathrm{N}} r^{2}} \int_{r_{0}=r_{\mathrm{C}}}^{r} \frac{1}{u_{\mathrm{I}, r}\left(r_{0}\right)} d r_{0},
$$

where $u_{\mathrm{I}, r}\left(r_{0}\right)$ is the velocity at $r$ of an ion produced at $r_{0}$. The energy gain by acceleration along the electric field (Equation (14)) results in

$$
u_{\mathrm{I}, r}\left(r_{0}\right)=\sqrt{u_{\mathrm{N}}^{2}+\frac{2 k_{B} T_{\mathrm{e}}}{m_{\mathrm{I}}} \ln \left(\frac{r}{r_{0}}\right)} .
$$

By inserting Equation (18) into Equation (17) and computing the integral, we obtain

$$
n_{\mathrm{I}, A F}(r)=\frac{Q f e^{\eta} \sqrt{\eta}}{4 \sqrt{\pi} u_{n}^{2} r}\left(\operatorname{erf} \sqrt{\eta+\ln \left(\frac{r}{r_{\mathrm{C}}}\right)}-\operatorname{erf} \sqrt{\eta}\right),
$$

where erf is the error function and where $\eta$ is a dimensionless parameter given by

$$
\eta=\frac{m_{\mathrm{I}} u_{n}^{2}}{2 k_{B} T_{\mathrm{e}}}
$$

For typical values of $u_{\mathrm{N}}$ and $T_{\mathrm{e}}$ the value of $\eta$ and its square root are $\ll 1$ such that

$$
\operatorname{erf} \sqrt{\eta} \approx \frac{2}{\sqrt{\pi}} e^{-\eta} \sqrt{\eta}
$$

and

$$
e^{-\eta} \approx e^{\eta} \approx 1
$$

Applying the point-source treatment $\left(r_{\mathrm{C}} \rightarrow 0\right)$,

$$
\lim _{r_{\mathrm{C}} \rightarrow 0} \operatorname{erf} \sqrt{\eta+\ln \left(\frac{r}{r_{\mathrm{C}}}\right)}=1 .
$$

By combining Equations (19) and (20)-(22), we get the relation

$$
n_{\mathrm{I}, A F *}(r)=\frac{Q f \sqrt{\eta}}{4 \sqrt{\pi} u_{n}^{2} r}\left(1-\frac{2}{\sqrt{\pi}} \sqrt{\eta}\right)=\frac{Q f_{\mathrm{H}_{2} \mathrm{O}}}{4 \pi u_{n}^{2} r}(\sqrt{\pi \eta}-2 \eta),
$$

and so we recover the $1 / r$ relation, although with a typically considerably lower plasma density than in the field-free case. This can also (see Equation (13)) be expressed as

$$
n_{\mathrm{I}, A F *}(r)=n_{\mathrm{I}, \mathrm{FFCF} *}(r)(\sqrt{\pi \eta}-2 \eta) .
$$

\section{REFERENCES}

Altwegg, K., Balsiger, H., Bar-Nun, A., et al. 2015, Sci, 347, 1261952 Balsiger, H., Altwegg, K., Bochsler, P., et al. 2007, SSRv, 128, 745 Bhardwaj, A. 2003, GeoRL, 30, 2244

Burch, J. L., Goldstein, R., Cravens, T. E., et al. 2007, SSRv, 128, 697 Cravens, T. E., Gombosi, T. I., Gribov, B. E., et al. 1984, Role of Electric Fields in the Cometary Environment, Rep. KFKI-1984-41 (Budapest, Hungary: Hungarian Acad. Sci.), 47

Cravens, T. E., Kozyra, J. U., Nagy, A. F., et al. 1987, JGR, 92, 7341

Edberg, N. J. T., Eriksson, A. I., Odelstad, E., et al. 2015, GRL, 42, 4263

Eriksson, A. I., Boström, R., Gill, R., et al. 2007, SSRv, 128, 729

Fuselier, S. A., Altwegg, K., Balsiger, H., et al. 2015, A\&A, in press

Goldstein, R., Burch, J. L., Mokashi, P., et al. 2015, GRL, 42, 3093

Gulkis, S., Allen, M., von Allmen, P., et al. 2015, Sci, 347, 0709

Hall, L. A., \& Hinteregger, H. E. 1970, JGR, 75, 6959

Hansen, K. C., Bagdonat, T., Motschmann, U., et al. 2007, SSRv, 128, 133

Haser, L. 1957, BSRSL, 43, 740

Hässig, M., Altwegg, K., Balsiger, H., et al. 2015, Sci, 347, 0276

Hill, T. W., Thomsen, M. F., Tokar, R. L., et al. 2012, JGR, 117, A05209

Hinteregger, H. E. 1981, AdSR, 1, 39

Huebner, W. F., Keady, J. J., \& Lyon, S. P. 1992, Ap\&SS, 195, 1

Huebner, W. F., \& Mukherjee, J. 2015, P\&SS, 106, 11

Khabibrakhmanov, I. K., \& Summers, D. 1997, JGR, 102, 2193

Koenders, C., Glassmeier, K.-H., Richter, I., Motschmann, U., \& Rubin, M 2013, P\&SS, 87, 85

Koenders, C., Glassmeier, K.-H., Richter, I., Ranocha, H., \& Motschmann, U. 2015, P\&SS, 105, 101

Körözmezey, A., Cravens, T. E., Gombosi, T. I., et al. 1987, JGR, 92, 7331

Lean, J. 1987, JGR, 92, 839

Lindsay, B. G., Sieglaff, D. R., Smith, K. A., \& Stebbings, R. F. 1997, PhRvA, 55,3945

Mada, S., Hida, K., Kimura, M., et al. 2007, PhRvA, 75, 022706

Mendis, D. A., Hill, J. R., Houpis, H. L. F., \& Whipple, E. C. 1981, ApJ, 249, 787

Morooka, M. W., Wahlund, J.-E., Eriksson, A. I., et al. 2011, JGR, 116, A1221

Nilsson, H., Stenberg-Wieser, G., Behar, E., et al. 2015, Sci, 347, 0571 
Rubin, M., Koenders, C., Altwegg, K., et al. 2014, Icar, 242, 38

Schunk, R. W., \& Nagy, A. F. 2009, Ionospheres: Physics, Plasma Physics and Chemistry (Cambridge: Cambridge Univ. Press)

Sierks, H., Barbieri, C., Lamy, P. L., et al. 2015, Sci, 347, 1044

Snodgrass, C., Tubiana, C., Bramich, D. M., et al. 2013, A\&A, 557, A33

Tenishev, V., Combi, M., \& Davidsson, B. 2008, ApJ, 685, 659
Trotignon, J. G., Michau, J. L., Lahoutte, D., et al. 2007, SSRv, 128, 713

Vigren, E., \& Galand, M. 2013, ApJ, 772, 33

Vigren, E., Galand, M., Lavvas, P., Eriksson, A. I., \& Wahlund, J.-E. 2015, ApJ, 798, 130

Woods, T. N., Eparvier, F. G., Bailey, S. M., et al. 2005, JGR, 110, A01312 